\title{
The Simulation of Energy Distribution of Electrons Detected by Segmental Ionization Detector in High Pressure Conditions of ESEM
}

\author{
V. Neděla, I. Konvalina, M. Oral and J. Hudec \\ Institute of Scientific Instruments ASCR, v.v.i, Brno, Czech Republic
}

\begin{abstract}
This paper presents computed dependencies of the detected electron energy distribution on the water vapour pressure in an environmental scanning electron microscope obtained using the EOD software with a Monte Carlo plug-in for the electron-gas interactions. The software GEANT was used for the Monte Carlo simulations of the beam-sample interactions and the signal electron emission from the sample into the gaseous environment. The simulations were carried out for selected energies of the signal electrons collected by two electrodes with two different diameters with the voltages of $+350 \mathrm{~V}$ and 0 , respectively, and then 0 and $+350 \mathrm{~V}$, respectively, and for the distance of $2 \mathrm{~mm}$ between the sample and the detection electrodes of the ionization detector. The simulated results are verified by experimental measurements. Consequences of the simulated and experimental dependencies on the acquisition of the topographical or material contrasts using our ionization detector equipped with segmented detection electrode are described and discussed.
\end{abstract}

\section{Introduction}

Environmental scanning electron microscope (ESEM) is indispensable for many experimental uses, including dry and electrically non-conductive samples. Apart from the compensation of the negative charge by positive ions from electron-gas interactions, ESEM allows studying fully hydrated, mostly biological samples at high pressures (units to thousands $\mathrm{Pa}$ ) [1], semiliquid or liquid samples as well as sample changes and reactions during dynamical in-situ experiments [2]. Danilatos claimed [3] that the image resolution in a high pressure in the ESEM can be comparable to that in the conventional scanning electron microscope (SEM). The resolution in ESEM is limited due to beam scattering, and also the signal-to-noise ratio in the detected signal is lower, but it can be mitigated by various adjustments: a decrease in the path length of the primary electrons (PE) through the high pressure region, an increase in the $\mathrm{PE}$ accelerating voltage and the probe current, a lower scanning speed or a suitable choice of the gas and its pressure.

Nevertheless, the high pressure conditions support amplification and detection of low energy signal electrons [4]. The detection principle is based on gas ionization which proceeds in a cascade between a grounded specimen holder and positively biased electrodes, commonly placed under the pole piece of the objective lens [5-7]. So far almost all detectors for ESEM use an electrostatic field to accelerate electrons and, consequently, a higher ionization of gas molecules, generation of signal electrons and positive ions, and increased signal amplification. Amplification of the detected signal is strongly influenced by the strength of the electric field between the grounded sample and the detection electrode as well as by the pressure and the type of gas in the specimen chamber of the ESEM. It was found that the optimal conditions are created by the presence of water vapour in the specimen chamber of ESEM [8].

The first commercial detector for ESEM based on this amplification phenomenon was called the environmental secondary detector (ESD) [9]. Unfortunately, the original version of the detector records a signal which is a mixture of SEs and backscattered electrons (BSEs); therefore the image is composed of SE topographical and BSE material contrasts [10]. For this reason the ESD was modified into the gaseous secondary electron detector (GSED) by using a suppressor electrode which allows detecting a cleaner SE signal [10]. Danilatos proposed a multi-electrode configuration of the 
detector to separate various detected signals [9]. The physical principle of a multi-electrode configuration was implemented into a technical design by Knowles and was used commercially in an ESEM by the FEI Company. Meredith et al. simulated contribution of PEs, BSEs and SEs to the total amplification from the ionisation [11]. Thiel et al. calculated the signal-to-background ratio for amplification of SEs in the GSED and the ESD [12]. Fletcher published curves showing a lower contribution of BSEs to the gas cascade amplified electron signal collected using the GSED as a function of the gas type and pressure in comparison with the ESD [10].

Some methods for the detection of separated SE and BSE signal have been examined also by our team. Autrata et al. designed a very sensitive BSE detector using the original YAG (yttrium aluminium garnet) scintillator for recording the material contrast [13]. The so-called combined ionization detector capable of detecting BSEs in SEM and ESEM by scintillation and SEs in ESEM by electron-gas ionization was realised $[14,15]$. If the specimen surface is to be observed in a topographical contrast, the ionisation function of the combined detector can be used. For this reason, a thin electrode is deposited on the bottom base of the YAG scintillator. If the electrode is at a positive voltage, the signal electrons initiate an ionisation cascade with gas molecules which amplifies the signal. Even if this detector was primarily designed as the SE detector for topographical observation a portion of BSE background is still detected. The small hole in the YAG scintillator can simultaneously serve as a second pressure limiting aperture (PLA 2) of the ESEM, an arrangement utilized in our AQUASEM II microscope [15]. The length of the high pressure region between the PLAs is decreased, and the diffusion of the electron beam in the differentially pumped chamber is minimised for this configuration.

The main objective of this work is to simulate signal electron trajectories for selected energies from several $\mathrm{eV}$ to tens of $\mathrm{keV}$ in gaseous environment for a specially designed ionisation detector equipped with two circular electrodes in order to better understand the connection between the configuration of the detection electrode or their segmented parts and the type of the detected information. The EOD program extended with our Monte Carlo module for electron-gas interactions and signal amplification is used to simulate trajectories of the signal electrons from a realistic sample model [4]. That is followed by an analysis of the energy distribution of the detected electrons by the detection electrodes.

In this paper, simulations of the detection efficiency for various conditions, detector configurations and types of detected electrons are presented. Simulated results are compared and verified by experiments, based on the assumption that the topographical contrast created by SEs can be observed using the detection electrode closest to the optical axis of the ESEM column. The material contrast, created by BSEs, is partially suppressed. On the other hand, a predominantly material contrast is detected by the detection electrode with a higher distance from the ESEM optical axis.

\section{Material and Methods}

The emission of the signal electrons from the gold surface was simulated using the GEANT Monte Carlo software [16] for 1000000 particles, the energy of the primary beam was $20 \mathrm{keV}$. The trajectories of the signal electrons emitted from the sample to water vapour environment between the sample and the segmented ionization detector and the signal amplification of the detected electrons were calculated using the EOD [17] (Electron Optical Design) software equipped with a Monte Carlo plug-in [4]. 10000 signal electrons with the energies of 1.7, 2.7, 4.7, 7.6, 10, 20, 50, 100, 300, $500,900,1000,2000,3000,5000,7500,10000,15000$ and $20000 \mathrm{eV}$ were traced through a $2 \mathrm{~mm}$ thick water vapor region (the sample to detection electrode distance) with the pressure of 50, 100, 200, 300, ..., $1000 \mathrm{~Pa}$. 
The electrons detected by the individual segments of the detector provide information about their final energy. The distribution of the electrostatic fields between the detector and the specimen, the spatial distribution of the signal electron emission from the sample as well as the gas type and the pressure all have a significant influence on the detected type of the signal electrons.

All experiments were made using our non-commercial experimental microscope, the AQUASEM-II [18]. The custom-built segmented ionization detector of secondary electrons with two ring detection electrodes was used for the detection of the signal electrons. The first electrode B with the inner diameter of $1.5 \mathrm{~mm}$ and the outer diameter of $4.5 \mathrm{~mm}$ was situated close to the optical axis of the ESEM, whereas the second electrode A with the inner diameter of $16 \mathrm{~mm}$ and the outer diameter of $20 \mathrm{~mm}$ was situated as far as possible from the microscope optical axis (Figures 1A, 1C). The detection system was made from 2 mm thick FR-4 copper clad laminate board. The electrodes A and $\mathrm{B}$ were made by etching and connected to a signal preamplifier. The detection system also has a small hole $(600 \mu \mathrm{m}$ in diameter, with its surface electrically conductive and grounded) situated in the centre of the detection system. The detection system simultaneously acts as the PLA 2 of the ESEM AQUASEM II [15].
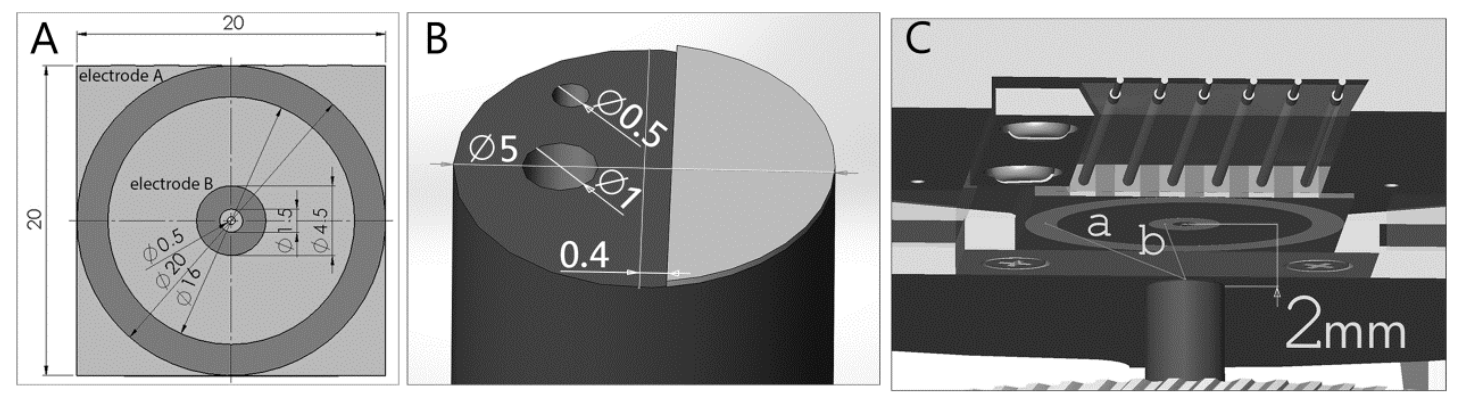

Figure 1. A: Detection electrodes of ionization detector for ESEM, B: Experimental sample consisting with two holes and gold plate, C: Experimental setup in ESEM AQUASEM II.

Two configurations of the detection system were used for the experiment. First, the positive potential of $350 \mathrm{~V}$ was applied on electrode A while electrode B was grounded. Second, the positive potential of $350 \mathrm{~V}$ was applied on electrode B and electrode A was grounded. An application of a positive bias of $350 \mathrm{~V}$ on the electrodes of the presented detection system acts similarly as the ESD. The detector is relatively simple both to construct and to model in simulations, therefore it was selected for a comparison of numerical results with experimental data and to help understanding the detection principles based on electron-gas interactions in ESEM.

The testing sample was made from a carbon rod ( $5 \mathrm{~mm}$ diameter, $8 \mathrm{~mm}$ width) with two holes and a small gold plate (Figure1B). An eccentrically situated hole $(0.5 \mathrm{~mm}$ diameter, $6 \mathrm{~mm}$ deep $)$ was used as the Faraday cup. The probe current was measured at the pressure of $0.01 \mathrm{~Pa}$ using the picoampermeter Keithley 485 before each experiment. Another hole (1 mm diameter, $6 \mathrm{~mm}$ deep) was used for the measurement of the background signal induced by beam diffusion in gas. Dependences of detected signal from gold on the gas pressure were measured (Figure 3B, dashed line) and also this signal with subtracted background (recorded by electrode A or B from the Faraday cup) was calculated and plotted (Figure 3B, full line).

The following experimental conditions were set: the beam accelerating voltage of $20 \mathrm{kV}$, the beam current of $25 \mathrm{pA}, 2 \mathrm{~mm}$ sample to detection electrode distance. For the experimental comparison a sample containing three metals with different atomic numbers and, therefore, different SE and BSE emission coefficients, was used. The three elements were mixed in alloys using different ratios, and 
these alloys are then in different areas on the sample - areas I, II and III, see Figure 4A. An energy dispersion X-ray analysis revealed that area I contains $57 \%$ of $\mathrm{Al}, 1 \%$ of $\mathrm{Cr}$ and $42 \%$ of $\mathrm{Pd}$, area II contains $70 \%$ of $\mathrm{Al}, 1 \%$ of $\mathrm{Cr}$ and $29 \%$ of $\mathrm{Pd}$, and area III contains $74 \%$ of $\mathrm{Al}, 17 \%$ of $\mathrm{Cr}$ and $9 \%$ of $\mathrm{Pd}$. The signal at the electrodes $\mathrm{A}$ and $\mathrm{B}$ from the three areas were determined from a line scan (Fig. 4A, B). The beam accelerating voltage was $20 \mathrm{kV}$, the probe current had a value of $120 \mathrm{pA}$, the sample to PLA distance was $2 \mathrm{~mm}$ and the water vapor pressure was $500 \mathrm{~Pa}$.

\section{Results}

The simulation consisted of two steps. First, the spectrum of the signal electrons was computed by the Monte-Carlo method in the Geant. Second, a dependence of the amplification coefficient on the energy and the pressure was obtained in the EOD (Figure 2). For each pressure, this dependence was used as a weight function in the integration of the signal electron emission spectrum, providing a value of the signal amplification coefficient for signal electrons of any emission energy (Figure 3A). The influence of the primary beam scattering was not considered in the simulations, as that would make them significantly even more time consuming.

The simulated results of the signal electrons at selected energies in a gas show that electrodes A and B collect signal electrons in the whole emission spectrum, recording a mixed contrast. Electrode B detects a higher number of low-energy electrons (Figures 2B, 2D), whereas electrode A with a higher diameter predominantly detects backscattered electrons, especially for the pressure of $300 \mathrm{~Pa}$ and higher (Figures 2A, 2C). All the results indicate that the proportion of the high-energy electrons increases with an increasing pressure. Multiplying the signal amplification in the gas by the simulated emission coefficients at a given energy gives the detection efficiency as a function of the initial energy of the signal electrons (Figures 2C, 2D). These dependencies help understanding contrast formation in the images in Figure 4, that were obtained using electrodes A and B and by evaluating differences between them.

The results were verified with an experiment. Using electrodes A and B the image of the sample was acquired for the three areas with different alloy ratios (Figure 4). The measured difference in the detected signal at the water vapour pressure of $500 \mathrm{~Pa}$ from electrode A from area II (point L1) and area I (point L3) is almost by $50 \%$ higher that the corresponding difference from electrode B under the same conditions (Figures 4A, 4B). The signal from electrode A contains a higher BSE contribution; BSE yield is higher for higher atomic numbers. To verify these conclusions, the sample was examined under the same conditions in vacuum in the Jeol JSM 6700F SEM, using a BSE-YAG detector which detects just BSEs (Figure 4C), and using an in-lens SE detector which obtains just the topographical contrast (Figure 4D). The signal from area II was calibrated for the same level during the experiments (Figures 4A-D).

The shift in the signal intensity maxima from electrode A towards higher pressure values compared to electrode B (Figures 3A and 3B) is caused mainly by a higher average length of the signal electron trajectories. The distances from the sample centre to the electrodes A and B are $9.22 \mathrm{~mm}$ and 2.50 $\mathrm{mm}$, respectively. The initially low-energy signal electrons are more influenced by the electrostatic field and collisions are more probable. Their trajectories then can be significantly longer.

Figures $3 \mathrm{~A}$ and $3 \mathrm{~B}$, dashed line, show a good agreement between the curve simulated with EOD software and experimental data. Computed and experimental results considerably depend on the geometry and the parameters of the detection system as well as on the geometry of the specimen chamber of the microscope. 
A ELECTRODE A - signal amplification in gas (simulated data)

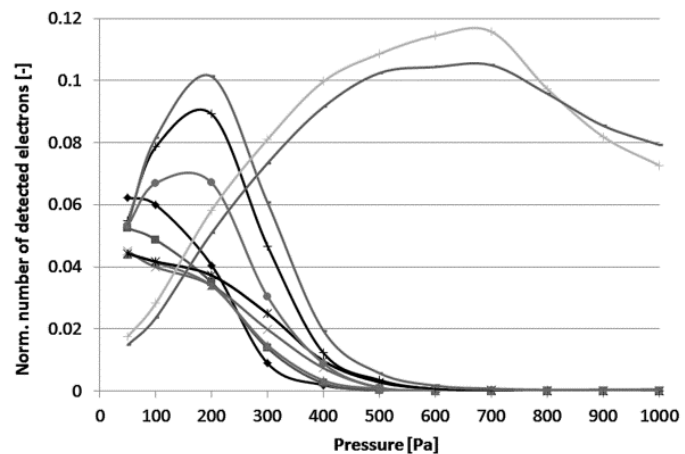

ELECTRODE A - signal amplification in gas (simulated data)

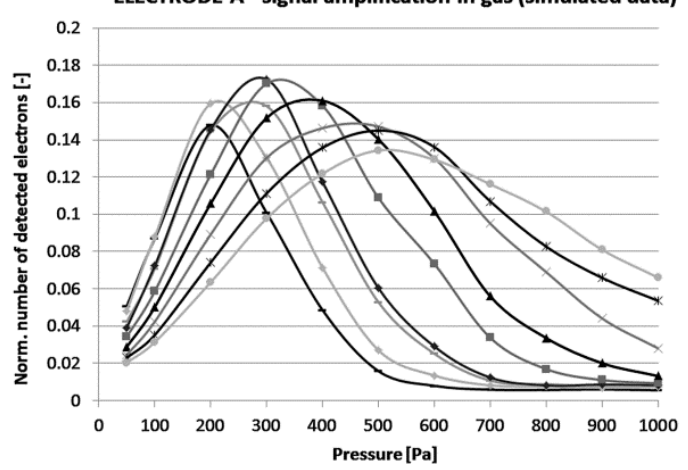

C ELECTRODE A - simulated with respect to energy distribution of

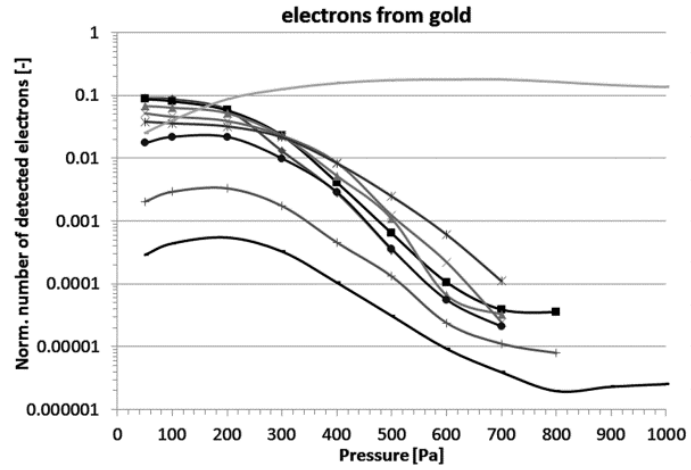

ELECTRODE A - simulated with respect to energy distribution of

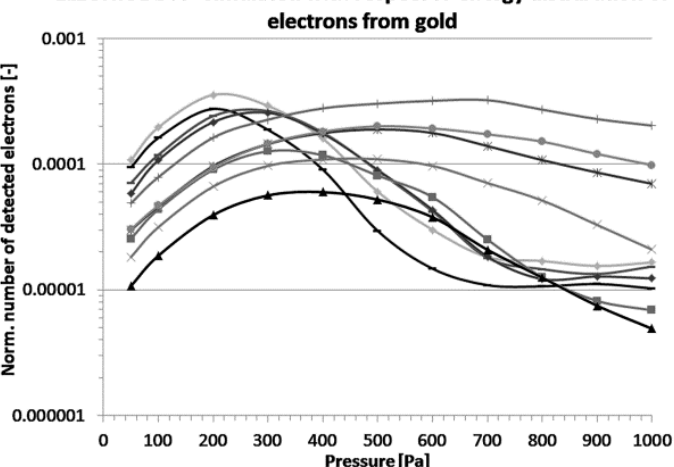

B $\rightarrow-1.7 \mathrm{ev}$
$-2.7 \mathrm{eV}$
$-4.7 \mathrm{ev}$
$-7.6 \mathrm{ev}$
$-10 \mathrm{ev}$
$-20 \mathrm{ev}$
$+50 \mathrm{ev}$
$-100 \mathrm{ev}$
$+15000 \mathrm{ev}$
$-20000 \mathrm{ev}$

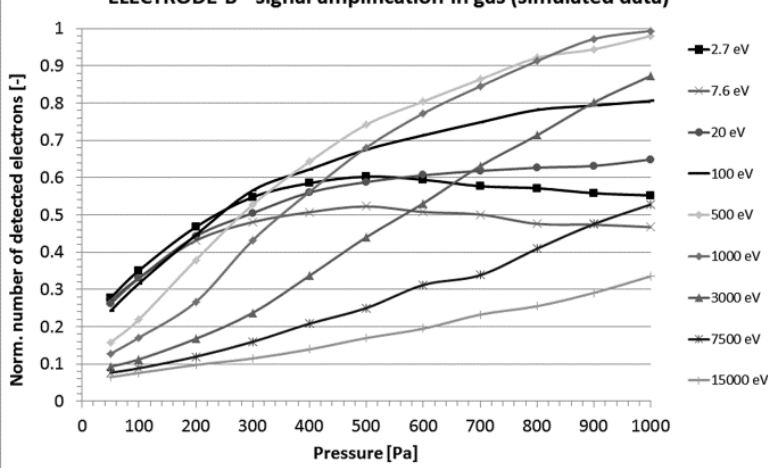

ELECTRODE B - signal amplification in gas (simulated data)

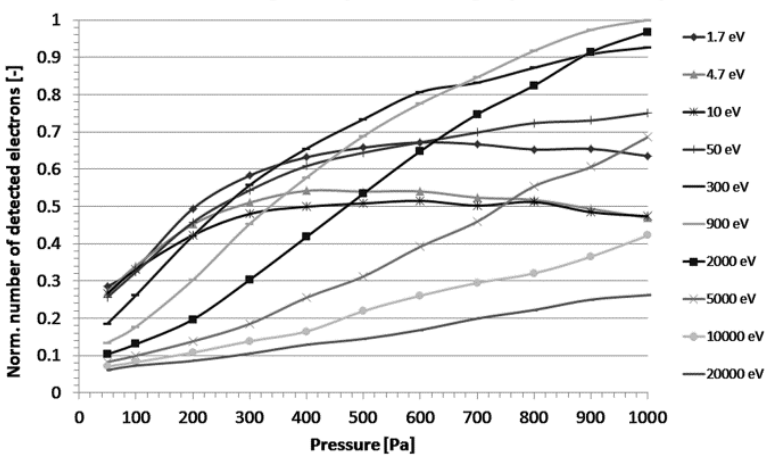

D ELECTRODE B - simulated with respect to energy distribution of

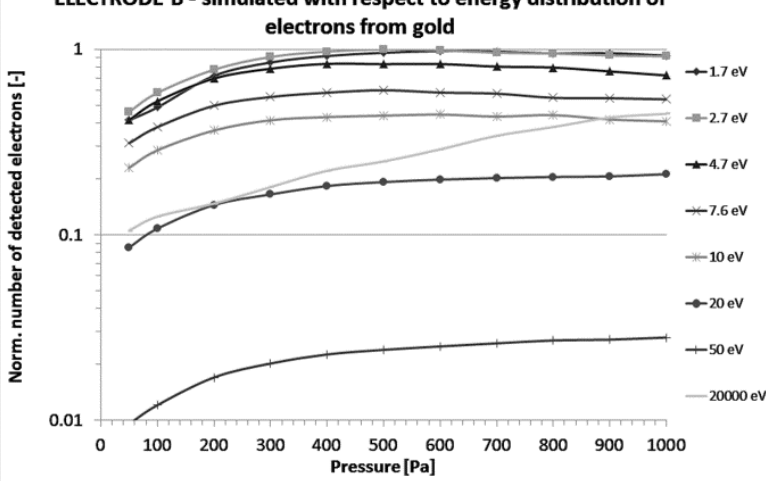

ELECTRODE B - simulated with respect to energy distribution of electrons from gold
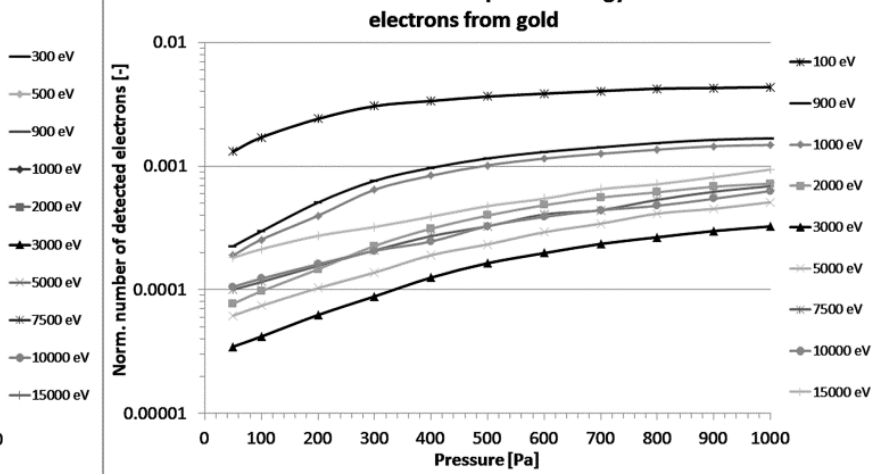

Figure 2. The dependence of the normalized number of electrons detected by electrodes A and B, see Figure1, on the water vapor pressure and the energy of the signal electrons using a gold sample. 

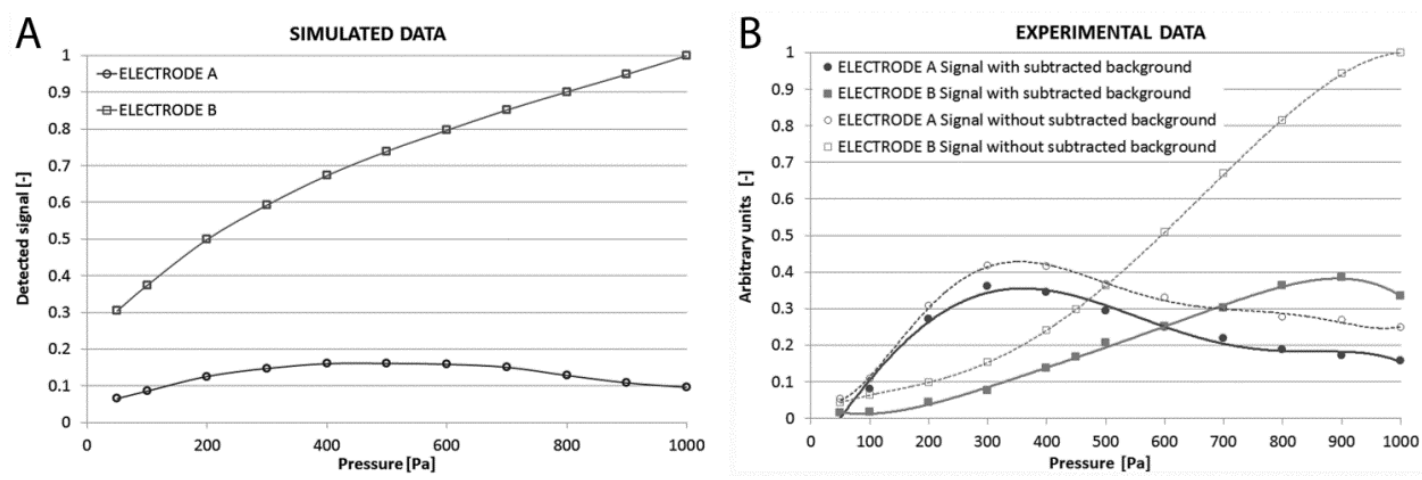

Figure 3. The dependence of the detected signal calculated by the EOD program (A) and obtained by the experiment (B) on the water vapour pressure; acc. voltage $20 \mathrm{kV}$; probe current $15 \mathrm{pA}$, sample to PLA distance $2 \mathrm{~mm}$, ionization detector at $350 \mathrm{~V}$ for detection electrodes $\mathrm{A}$ and $\mathrm{B}$, see Figure 1

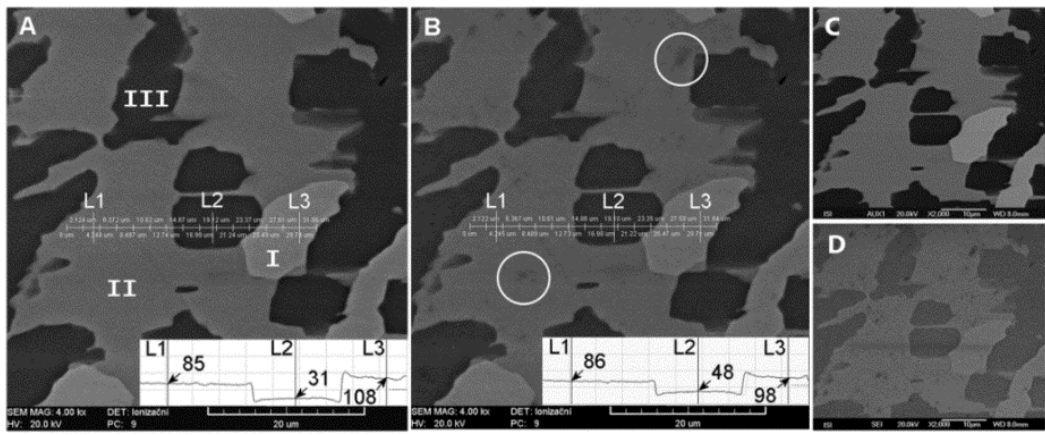

Figure 4. Three types of materials with different Z (I,II,III); A: using electrode A, B: using electrode $\mathrm{B}$ in ESEM, ionization detector, acc. voltage $20 \mathrm{keV}$, vapor pressure $500 \mathrm{~Pa}$. C: using BSE-YAG detector, D: using in-lens SE detector in Jeol JSM 6700F; acc. voltage $20 \mathrm{keV}$, vacuum.[19]

References:

[1] V Neděla, Microscopy Research Technique 70 (2007), p. 95.

[2] AM Donald, Nat. Mater. 2 (2003), p.511.

[3] GD Danilatos, J. Microsc. 160 (1990), p.9.

[4] V Neděla et al, Nucl. Instrum. Meth. A 645 (2011), p. 79.

[5] GD Danilatos, Microsc. Microanal. 6 (2000), p. 12.

[6] M Toth et al, Ultramicroscopy 94 (2003), p. 71.

[7] J Jirák et al, J. Microsc. 239 (2010), p. 233.

[8] AL Fletcher et al, J.Phys. D: Appl.Phys. 30 (1997), p. 2249.

[9] US Patent No. 4,897,545.

[10] AL Fletcher et al, J. Microsc. 196 (1999), p.26.

[11] P Meredith et al, Scanning 18 (1996), p.467.

[12] BL Thiel et al, J. Microsc. 187 (1997), p.143.

[13] R Autrata et al, Scanning 14 (1992), p.127.

[14] V Romanovský et al, European Microscopy and Analysis 59 (1999), p. 19.

[15] V Neděla et al, Microsc. Microanal. 17 (S2) (2011), p. 920.

[16] S Agostinelli et al, Nucl. Instr. Meth. A 506 (2003), p. 250.

[17] B Lencová et al, Phys. Procedia 1 (2008), p.315.

[18] V Neděla, J. Microsc. 237 (2010), p. 7.

[19] This work was supported by the Grant Agency of the Czech Republic, grant No. GA14-22777S. 